Volume 8. No. 9, September 2020

International Journal of Emerging Trends in Engineering Research

Available Online at http://www.warse.org/IJETER/static/pdf/file/ijeter136892020.pdf

https://doi.org/10.30534/ijeter/2020/136892020

\title{
Trainable WEKA Segmentation of Retinal Fundus Images for Global Eye Disease Diagnosis Application
}

\author{
Vijayan $T^{1}$, Sangeetha $M^{2}$, Karthik $B^{3}$ \\ ${ }^{1}$ Research scholar, Electronics and Communication Engineering, BIST, Bharath Institute of Higher Education \\ and Research, Chennai, India. tvij16@gmail.com \\ ${ }^{2}$ Professor, Electronics and Communication Engineering, BIST, Bharath Institute of Higher Education and \\ Research, Chennai, India. sang_gok@yahoo.com \\ ${ }^{3}$ Associate Professor, Electronics and Communication Engineering, BIST, Bharath Institute of Higher Education \\ and Research, Chennai, India. karthikguru33@gmail.com.
}

\begin{abstract}
Image segmentation is a challenging task and quite important in biomedical images for disease identification and measuring the progress of the disease. Segmentation and extraction of retinal blood vessels, micro aneurysms, hemorrhages, soft exudates and hard exudates have a significant attention to diagnosis various retinal related diseases especially diabetic retinopathy and glaucoma. The proposed work effectively segments the above-mentioned regions from the retinal images with Contrast Limited Adaptive Histogram Equalization (CLAHE) preprocessing and Fast Random Forest Classifier supported by Trainable WEKA segmentation of FIJI Tool. The proposed work segments each and every pixel of the retinal image into predefined classes and the produced good segmentation results as shown in the paper.
\end{abstract}

Key words: Diabetic retinopathy, Machine Learning, Semantic segmentation, Contrast Limited Adaptive Histogram Equalization, Fast Random Forest.

\section{INTRODUCTION}

In recent years diabetic retinopathy (DR), is a chronical eye disease among diabetic patients who have more than 15 years of diabetes with uncontrolled blood sugar grabbed the attention of researchers. Early detection and treatment planning for diabetic retinopathy prevent the vision loss and other serious complications. DR broadly classified into two main categories: (i) Non-Proliferative diabetic Retinopathy (NPDR) and Proliferative Diabetic retinopathy (PDR). Diabetic retinopathy affects eye visions gradually, as a starting stage symptom it develops blurred vision, thickness of the retinal valve, impairment of colour vision, patches or streaks that block the person's vision are other related symptoms and advanced stage symptoms indicated by haemorrhages and irregular progress of new blood vessels.
Diabetic retinopathy was diagnosed by physical examination of retina and retinal fundus image analysis by expert ophthalmologists. Proper identification and analysis of retinal blood vessels, micro aneurysms, hemorrhages, soft and hard exudates help to diagnose this disease and the proposed work will help to achieve this.

T. Jemima Jebaseeli et al (2019) [1] proposed work increases the image contrast based on Contrast Limited Adaptive Histogram Equalization (CLAHE) and implemented Tandem Pulse Coupled Neural Network (TPCNN) model for automatic feature generation and classification. Deep Learning Based Support Vector Machine (DLBSVM) method used for extraction of the retinal blood vessels. Donghuan Lu et al (2019) [2] proposed Multiclass fluid segmentation and detection in the retinal OCT images using deep learning algorithms. Random forest classifier was adopted to detect and discard the incorrectly labelled fluid regions from the segmented fluid regions. N Nur et al (2019) [3] followed three steps, namely optic disc removal, location detection of exudates, and exudates segmentation. For optical disc removal done by midpoint circle algorithm and exudate path segmentation based on saliency method. Ignacio Arganda-Carreras et al (2014) [4] presented Trainable Weka Segmentation (TWS) is a versatile tool for Microscopy Image segmentation based on pixel classification. TWS is working link among the machine learning algorithms and the Medical image package Communities. Muhammad Arsalan et al (2019) [5]: proposed Segmentation and diagnosis of DR using Vess-net based on Artificial intelligence.

The objective of the proposed work is to segment the optical discs, hemorrhages, retinal blood vessels and exudates from the retinal fundus images using Trainable WEKA segmentation (TWS) [6-7]. The input image is preprocessed using CLAHE techniques for better segmentation results and pixel wise classification to predefined classes was performed by Fast random forest classifier. TWS convert image pixel to 
Vijayan T et al., International Journal of Emerging Trends in Engineering Research, 8(9), September 2020, 5750 - 5754

numerical instances, each instances-based value segmentation progress done by machine learning classifier. Each same type of pixels is grouping in manual class [23-25].

In this paper flow work as follows. Section II describers' materials and methods towards DR image pre-processing and Segmentation classifier. Section III presents Trainable WEKA segmentation procedure. Section IV depicts the experimental results and discussion and TWS concludes in Section V.

\section{MATERIAL AND METHODS}

This section describes the pre-processing techniques, classifier, tool and procedures followed for segmenting the retinal fundus images. The criteria followed for class selection for the classification of pixels are also explained.

\subsection{Contrast Limited Adaptive Histogram Equalization (CLAHE)}

CLAHE [8] method is used for enhancing the local contrast of the input retinal fundus image. As a result, the visibility of the diabetic retinopathy identification signs like hemorrhages, retinal blood vessels, micro aneurysms, and exudates has improved in the pre-processed fundus images. The parameters of CLAHE function such as block size, histogram bins and max slope is changed and the results were observed and the best results were obtained for (Block size127, Histogram bins-256, and max slope-3.00).[9] This pre-processing technique improved the segmentation capability and classification accuracy of the model.

\subsection{Fast Random Forest (FRF) Classifier}

Fast Random Forest (FRF) classifier is the re-implementation structure of renowned Random forest algorithm by Waikato Environment for Knowledge Analysis (WEKA). FRF classifier is a very efficient supervised learning algorithm which is used for both segmentation and classification. The Random forest algorithm segments each and every pixel of the image features into numerical value based on multiple classification trees. The group of same pixels named such as classes [9].

\subsection{Trainable WEKA Segmentation}

Fiji[4-9] is an open source free software under the GNU General Public License and an image processing package- a "batteries-included" distribution of ImageJ, contains a comprehensive collection of source codes, libraries and plugins to facilitate analyses of digital images. The Trainable Weka Segmentation is a Fiji plugin used for pixel-based segmentations by combining machine learning algorithms with a set of selected image features. WEKA can itself be called from the plugin.
Easy to use graphical user interfaces, portability, accessibility of imagining tools and algorithms for data analysis and free availability are the reasons for selecting this tool for this application.

\section{TRAINABLE WEKA SEGMENTATION OF RETINAL FUNDUS IMAGES}

The flow graph for the proposed Trainable WEKA segmentation framework shown in Figure 1 describes the entire process involved in this segmentation. Data obtained from Kaggle [10] Diabetic Retinopathy public data set containing Diabetic retinopathy and non-diabetic retinopathy retinal fundus images. DR images were categorized by professional graders into two classes (NPDR and PDR) by proper observation of indicators. Kaggle public dataset's retinal fundus images were captured by different devices in multiple dimensions.[10]

Trainable Weka Segmentation [4] of retinal fundus image is obtained by the following steps:

a) Import DR image or DR image Sequence

b) Apply Enhance local contrast (CLAHE)

c) Select plugins---> Segmentation----> Trainable Weka Segmentation

d) Define classes for each identifier

e) Apply Fast Random Forest Classifier

First, the images are resized to an even 1024 *1024-pixel dimensions through proper cropping of the inner retina part and removing unwanted backgrounds. CLAHE pre-processing technique is applied to enhance the image contrast for easy identification of DR signs in the fundus image [8].

To define classes for the FRF classifier the sample pixels of each indicators such as Retinal blood vessels, haemorrhages (HEs), microaneurysms (MAs), soft exudates (SEs) and hard exudates are selected manually and assigned to specific class as shown in Table 1.[11-15] Pixels corresponding to each class are shown in different colour in Figure $2 \mathrm{~d}$. Indicators are traced with utmost care and repeated on multiple images for better segmentation results[16]. The application of CLAHE pre-processing technique on the retinal images gives clear view of indicators and made this sampling work easier and accurate [17].

Table 1: Pre-defined Class Vs DR Identification

\begin{tabular}{|l|l|}
\hline Diabetic Retinopathy Identification Signs & Pre-defined Class \\
\hline Retinal blood vessels and haemorrhages & Class 1 \\
\hline Optical disc & Class 2 \\
\hline Micro aneurysms and cotton wool spots & Class 3 \\
\hline Soft and hard exudates & Class 4 \\
\hline
\end{tabular}

The Fast Random Forest algorithm uses a large number of (default 200) classification trees for elective each pixel of the input image to a predefined classes or the 
Vijayan T et al., International Journal of Emerging Trends in Engineering Research, 8(9), September 2020, 5750 - 5754

indicators, with its equivalent set of features, belongs to. Random forest algorithm presents multiple decision trees, one's classifier is trained the retinal images, multiple sequence of same image done by equal function set of pixel-based segmentation. Enterier process convert pixel to numerical instances values. This segmentation process very clearly indicates the disease sign for further classification function $[9,16]$.

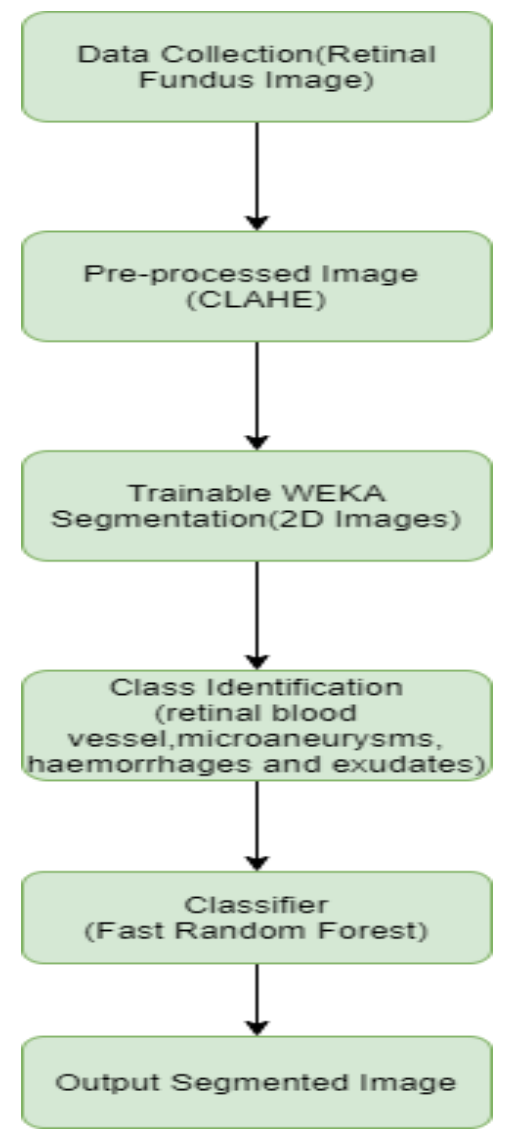

Figure 1: The proposed frame work for Trainable WEKA segmentation

\section{RESULT AND DISCUSSIONS}

The segmentation results show the selected CLAHE preprocessing technique and FRF classifier produced good segmentation results as shown in Figure 2. Fig $2 b$ is the CLAHE enhanced image of the original image Fig. 2a after preprocessing and shows clear view of the retinopathy identifiers. Manual selection of sample points for the identifiers is shown in Fig 2c.

Each identifier is defined as separate class as shown in Table 1. Fig $2 \mathrm{~d}$ and Fig. $2 \mathrm{e}$ shows the result of the Trainable WEKA segmentation trained by Fast Random Classifier and each class is represented with unique colors. Fig $2 \mathrm{f}-2 \mathrm{i}$ shows the probability maps of each classes and helps the user to easily understand the severity of DR in diabetic retinopathy detection applications [18-22].

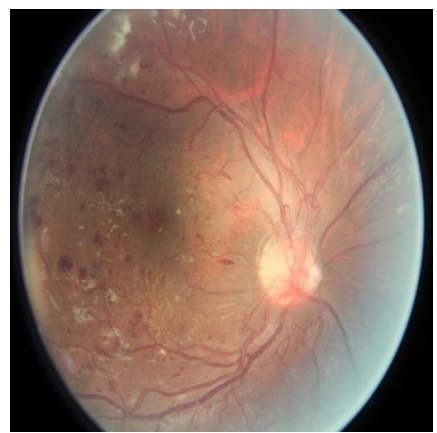

(a) Original DR image

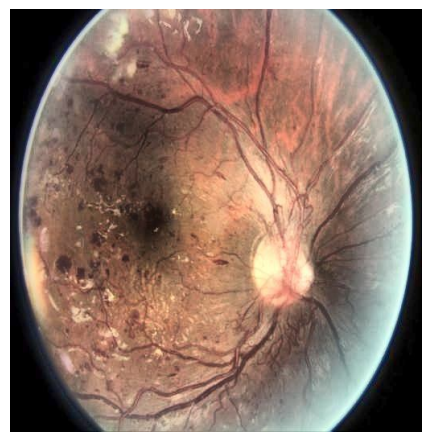

(b) CLAHE Image

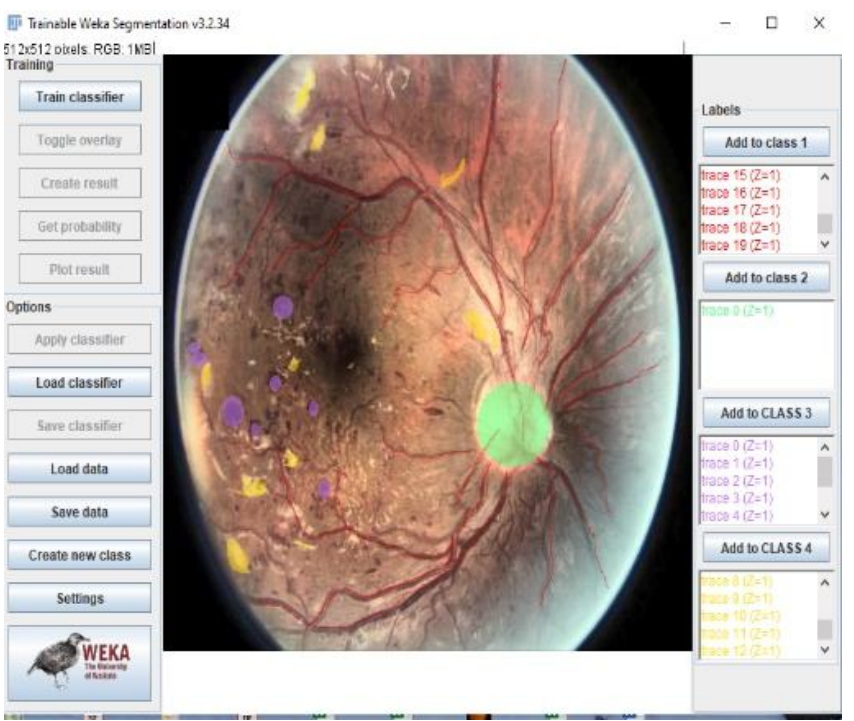

(c) DR image Manual class Marking (Fiji Tool)

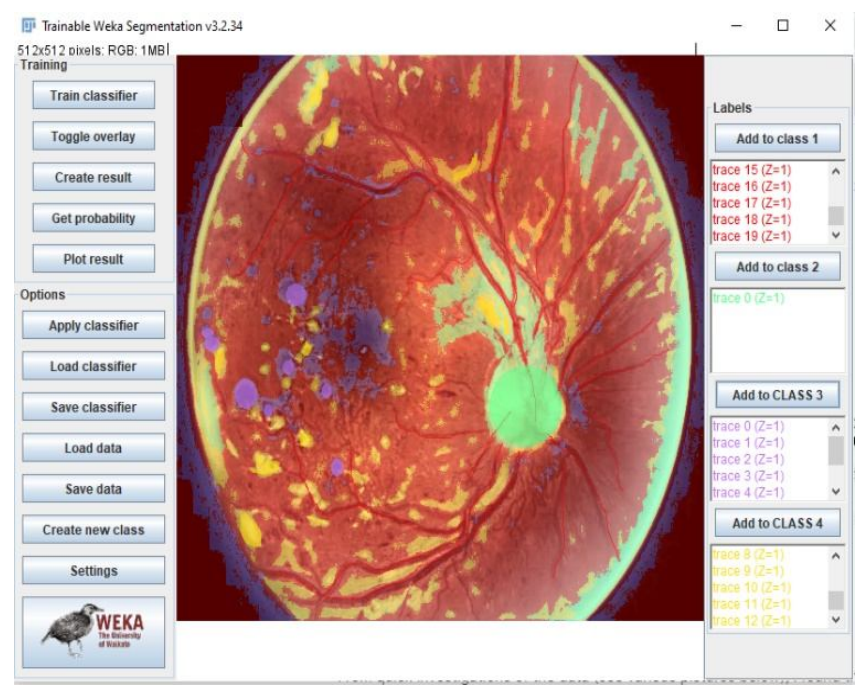

(d) Segmented DR Image after trained with FRF 


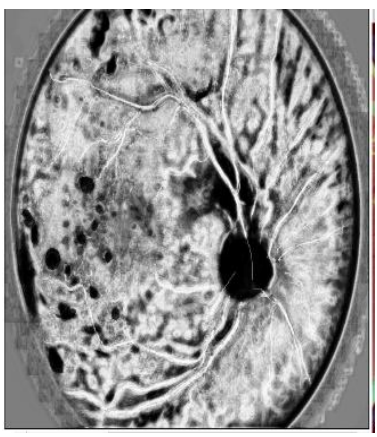

(e)

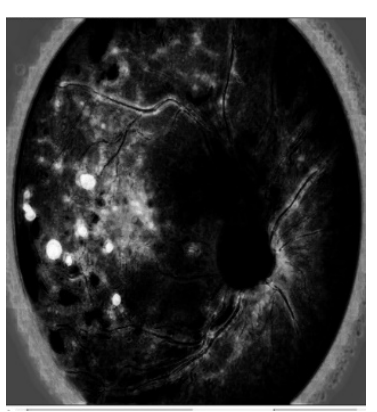

(h)

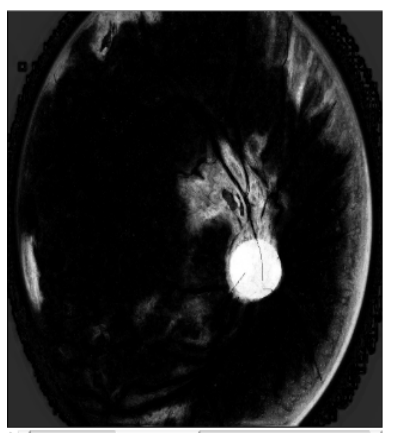

(f)

(g)

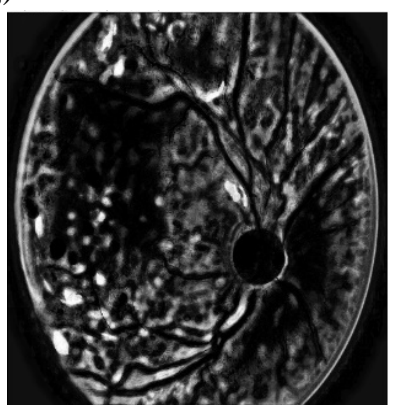

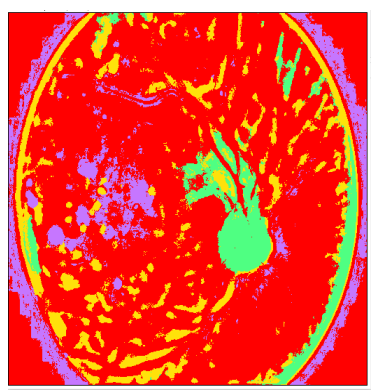

(i)

Figure 2: Illustration of TWS for Diabetic retinopathy Image: (a) original DR image, (b)Contrast Limited Adaptive Histogram Equalization Image,(c) DR Class Manual identification (Retinal blood vessels, MAs,HEs, and Exudates), (d)Train DR image (FRF classifier), (e)-(h) probability maps (Retinal blood vessels, optical disc, MAs, HEs, and Exudates),(i) Final TWS output.

This model is applied on various retinal fundus images and produced good segmentation results. The results show the segmentation output are closer to the ground truth.

\section{CONCLUSION}

The discussed Fast Random Forest Classifier based Trainable WEKA segmentation method will identify the diabetic retinopathy identification signs such as thickness of blood vessels, micro aneurysms, hemorrhages and exudates more effectively if the classes are defined properly. Quick labelling of classes, robustness and classification accuracy are

the reasons for opting this FRF based Trainable WEKA segmentation. The proposed work will support the retinal image-based decision-making tasks on diseases diagnosis like Diabetic Retinopathy, Glaucoma. Implementing this segmentation model as a pre-processing step may progress the classification accuracy in diabetic retinopathy applications. We are currently working in the above-mentioned task and getting comparable results over other traditional techniques.

\section{REFERENCES}

1. T. Jemima Jebaseeli, C. Anand Deva Durai, and J. Dinesh Peter. Segmentation of retinal blood vessels from ophthalmologic Diabetic Retinopathy images, Computers and Electrical Engineering, Vol.73, pp.245-258,2019

2. Donghuan Lu \& Morgan Heisler et al. Deep-learning based multiclass retinal fluid segmentation and detection in optical coherence tomography images using a fully convolutional neural network, Medical image Analysis, Vol.54, pp.100-110,2019.

3. N Nur \& H Tjandrasa et al. Exudate Segmentation in Retinal Images of Diabetic Retinopathy Using Saliency Method Based on Region, IOP Conf. Series: Journal of Physics: Conf. Series 1108 012110,2018.

4. Ignacio Arganda-Carreras and Verena Kaynig et al. Trainable Weka Segmentation: A Machine Learning Tool for Microscopy Image Segmentation, Arganda-Carreras, pp.74-80.2014.

5. Muhammad Arsalan and Muhammad Owais et al. Aiding the Diagnosis of Diabetic and Hypertensive Retinopathy Using Artificial Intelligence-Based Semantic Segmentation, J. Clin. Med., Vol.8, No. 1446, pp.1-27, 2019.

6. Wang S, Yin Y Cao G, Wei B, Zheng Y and Yang G. Hierarchical retinal blood vessel segmentation based on feature and ensemble learning, Neuro Comput, Vol.149, Part B, pp.708-17,2015.

7. Vega R, Sanchez-Ante G, Falcon-Morales LE, Sossa H and Guevara E. Retinal vessel extraction using Lattice Neural Networks with dendritic processing, Computers Biology and Medicine, Vol 58, pp.20-30,2015.

8. Feng Li \& Zheng Liu et al. Automatic Detection of Diabetic Retinopathy in Retinal Fundus Photographs Based on Deep Learning Algorithm, Trans Vis Sci Tech., Vol 8, No 6, pp 1-13,2019.

9. https://imagej.net/

10. Kaggle: https://www.kaggle.com/

11. Varun Gulshan, Lily Peng et al. Development and Validation of a Deep Learning Algorithm for Detection of Diabetic Retinopathy in Retinal Fundus Photographs, The Journal of the American Medical Association, 316(22), pp.2402-2410, 2016. 
Vijayan T et al., International Journal of Emerging Trends in Engineering Research, 8(9), September 2020, 5750 - 5754

12. C. Mahiba, and A. Jayachandran. Severity analysis of diabetic retinopathy in retinal images using hybrid structure descriptor and modified CNNs, Measurement, Vol.135, pp.762-767,2019.

13. Filippo Arcadu and Fethallah Benmansour et al. Deep learning algorithm predicts diabetic retinopathy progression in individual patients, npj Digital Medicine volume 2, Article number: 92, pp.1-9, (2019)

14. R. Karthikeyan \& P. Alli et al. Feature Selection and Parameters Optimization of Support Vector Machines Based on Hybrid Glowworm Swarm Optimization for Classification of Diabetic Retinopathy, Journal of Medical Systems,42(10):195, pp.1-11,2018.

15. AnilkumarB and P. Rajesh Kumar. Tumor Classification using Block wise fine tuning and Transfer learning of Deep Neural Network and KNN classifier on MR Brain Images, International Journal of Emerging Trends in Engineering Research, vol 8, No 2, pp. $769-775$, Feb 2020

16. Ashutosh Kumar and vijayan $\mathrm{T}$ et al. Glaucoma Detection Based on Optic Disc and Optic Cup Segmentation Using Feed Forward Neural Network, International Journal of Applied Engineering Research (IJAER), Vol 10, No. 6, pp.15857-15868,2015.

17. Sazak, Ç.; Nelson, C.J.; Obara, B.et al. The multiscale bowler-hat transform for blood vessel enhancement in retinal images, Elsevier, Pattern Recognit., vol 88, pp.739-750,2019.

18. Alam, M.; Le, D.; Lim, J.I.; Chan, R.V.P.; Yao, X. Supervised machine learning based multi-task artificial intelligence classification of retinopathies, $\mathrm{J}$. Clin. Med., Vol. 8(6):872, pp.1-15,2019.

19. Kranthi Kumar Palatalise and Bhavani Sambaturu. Automatic Diabetic Retinopathy Detection Using Digital Image Processing, International Conference on Communication and Signal Processing, April 3-5, India., pp.0072-0076, 2018.

20. Shailesh Kumar and Basant Kumar. Diabetic Retinopathy Detection by Extracting Area and Number of Microaneurysm from Colour Fundus Image,5th International Conference on Signal Processing and Integrated Networks (SPIN), pp.359-364,2018.

21. B. Wu, W. Zhu, F. Shi, S. Zhu, and X. Chen. Automatic detection of microaneurysms in retinal fundus images, Computerized Medical Imaging and Graphics, Vol. 55, pp. 106-112, 2017.

22. Christodoulides A, Hurtut T, Tahar HB and Cheriet F. A multi-scale tensor voting approach for small retinal vessel segmentation in high resolution fundus images, Comput Med Imaging Graph, Vol.52, pp.28-43,2016.

23. Rajesh Babu, $\mathrm{K}$ and $\mathrm{T}$. Manjula2. Brain Tumor Segmentation by Level-Set and Chan-Vese Methods using different Fusion Approaches, International Journal of Emerging Trends in Engineering Research, vol 8, No 3, pp. 769 - 775, Mar 2020
24. Sivachandar Kasiviswanathan, Thulasi Bai Vijayan, Lorenzo Simone and Giovanni Dimauro. Semantic Segmentation of Conjunctiva Region for Non-Invasive Anemia Detection Applications, Electronics 2020, 9, 1309; pp.1-13, Aug 2020 doi:10.3390/electronics9081309.

25. Pedro Costa, Adrian Galdran and Asim Smailagic. A Weakly-Supervised Framework for Interpretable Diabetic Retinopathy Detection on Retinal Images, Special Section on Advanced Signal Processing Methods in Medical Imaging, IEEE Access, vol 6, pp.18747-18758,2018. 